\title{
Characterization of Suicidal Behaviour with Self-Organizing Maps
}

\author{
José M. Leiva-Murillo, ${ }^{1}$ Jorge López-Castromán, ${ }^{2}$ \\ Enrique Baca-García, ${ }^{2}$ and EURECA Consortium ${ }^{3}$ \\ ${ }^{1}$ Department of Signal Theory and Communication, Universidad Carlos III de Madrid, Avenida Universidad 30, \\ 28911 Madrid, Spain \\ ${ }^{2}$ Department of Psychiatry, Fundación Jiménez Díaz, Avenida Reyes Católicos 2, 28040 Madrid, Spain \\ ${ }^{3}$ Centre Hospitalier Regional Universitaire de Montpellier, France
}

Correspondence should be addressed to José M. Leiva-Murillo; jose@tsc.uc3m.es

Received 30 November 2012; Revised 5 March 2013; Accepted 6 March 2013

Academic Editor: Reinoud Maex

\begin{abstract}
Copyright @ 2013 José M. Leiva-Murillo et al. This is an open access article distributed under the Creative Commons Attribution License, which permits unrestricted use, distribution, and reproduction in any medium, provided the original work is properly cited.

The study of the variables involved in suicidal behavior is important from a social, medical, and economical point of view. Given the high number of potential variables of interest, a large population of subjects must be analysed in order to get conclusive results. In this paper, we describe a method based on self-organizing maps (SOMs) for finding the most relevant variables even when their relation to suicidal behavior is strongly nonlinear. We have applied the method to a cohort with more than 8,000 subjects and 600 variables and discovered four groups of variables involved in suicidal behavior. According to the results, there are four main groups of risk factors that characterize the population of suicide attempters: mental disorders, alcoholism, impulsivity, and childhood abuse. The identification of specific subpopulations of suicide attempters is consistent with current medical knowledge and may provide a new avenue of research to improve the management of suicidal cases.
\end{abstract}

\section{Introduction}

Suicide is one of the leading causes of the Global Burden of Disease, accounting for approximately 1.5\% [1]. Every year, about 10 to 20 million people attempt suicide worldwide and one million people actually die because of selfinflicted harm (Data from the WHO, http://www.who.int/ mental_health/prevention/suicide). This fact is particularly alarming among the young; suicide causes $6.3 \%$ of the global deaths from 10 to 24 years of age [2], and this percentage can rise to $15 \%$ in high-income countries. However, suicidal behavior results from a complex interaction between vulnerability factors and environmental events [3], making it difficult to predict or prevent. Many risk and protective factors for suicidal behavior have been consistently identified [4], but predictive models remain imprecise [5]. A better understanding of the hierarchy and organization of variables involved in suicidal behavior may help to improve the detection of potential suicide victims.
Given the complexity of the problem, classical statistical methods are not able to appropriately deal with large sample sizes, high numbers of variables, and strong nonlinear interactions in the data. Modern machine learning and data mining approaches, on the other hand, overcome these limitations and have been successfully applied to computational biology problems such as suicide attempter classification [6]. If sequential data are also available, these methods are able to uncover patterns in the evolution of mental disorders [7].

One of the problems most intensively studied in machine learning is variable selection, which in our particular application consists in identifying the most relevant risk factors in suicidal behavior. Let $\mathbf{x}$ be a random variable so that a given dataset $\left\{\mathbf{x}_{\mathbf{i}}\right\}_{i=1, \ldots, L} \in \mathscr{R}^{D}$ consists of $L$ realizations of $\mathbf{x}$. Variable selection searches for a subset of variables among the $D$ original ones. Feature selection and dimensionality reduction are open problems in machine learning since they are subject to the no free lunch principle: a variable selection strategy cannot uniformly outperform other ones in terms of 
prediction ability $[8,9]$. In supervised learning, the variables must be selected according to their ability at predicting an auxiliary variable $y$, which in our case indicates whether the suicidal behavior is observed or not. Variable ranking is a simplification of the variable selection problem that consists in ordering the variables in decreasing order of relevance.

There is an inherent difficulty regarding feature selection: it is a problem with nonpolynomial complexity (i.e., an NPproblem) unless some particularity of the problem can be exploited. The reason is that all the possible combinations of variables must be evaluated in order to choose the optimal one. For this reason, most of the feature selection methods described in the literature are suboptimal, based on forward selection, backward elimination, or a branch-and-bound approach [10].

An additional difficulty arises when a particular outcome is linked to different subsets of variables depending on the population. This is the case of evaluating the risk of suicide behavior. For this reason, a successful method should be able to (i) cluster the population in a nonlinear way, (ii) use a criterion to find the relevant variables in each subpopulation, and (iii) allow for interacting with the practitioner in the selection of the most clinically relevant information from the different subpopulations.

In this paper, we propose to use a self-organizing map (SOM) to find nonlinear dependencies in the data [11]. Although the SOM is not a state-of-the-art technique in either supervised learning or variable selection, there are a number of reasons that can make it useful in this particular application. First, a SOM provides a simple visualization that facilitates the recognition of the data structure not only to the statistician, but also to the physician. Secondly, it shares some properties with state-of-the-art methods: it can deal with high-dimensional data and nonlinear patterns. Third, although SOMs are intended for unsupervised learning, they have been successfully applied to supervised problems such as bankruptcy prediction [12].

The presented work is novel from both the medical and the computational biology points of view. From the medical perspective, the study analyzes a large cohort of 8,699 subjects compiled by five institutions from four different countries that are involved in the EURECA consortium. (The European Research Consortium for Suicide is composed of research groups on suicide from Montpellier, Geneva, Molise, Oviedo, and Madrid.) Each subject has been characterized by 606 variables related to the sociodemographic status of the subjects, as well as their answers to normalized questionnaires that measure hostility, impulsivity, alcoholism, childhood trauma, hopelessness, and so forth. From the computational biology or artificial intelligence point of view, this is the first work, up to the authors' knowledge, that successfully performs a combination of a nonlinear unsupervised learning tool such as the SOM with linear discriminants for variable selection in a supervised learning setting.

In the next section, both the data under study and the method proposed for variable selection are described. In Section 3, we show the results together with the their interpretation from both the machine learning and the medical point of view. Section 4 closes the paper with the most relevant conclusions.

\section{Materials and Methods}

In this section, we first describe the dataset under study. Then, in Section 2.2, the elements from linear discriminants and SOMs that are involved in the study are described.

2.1. Dataset. The EURECA consortium have recruited 3,839 suicide attempters and completers over the last years [13]. These clinical teams implemented very similar clinical methods and assessment procedures. Sociodemographic and clinical data of the subjects have been joined in a common database together with the results of assessment tools. All suicide attempters have been hospitalized following an attempt that was defined as follows: "a potentially self-injurious behavior with a nonfatal outcome, for which there is evidence (either explicit or implicit) that the person intended at some (nonzero) level to kill himself/herself." This definition has been adopted by the National Institute of Mental Health (NIMH) and the main research groups in the UE [14]. Studies were approved by the research ethics committee of each group and conducted according to the tenets of the Declaration of Helsinki. All participants signed an informed consent form after the explanation of the study objective and procedures. In addition to suicide attempters, the dataset includes psychiatric patients with no records of suicide attempts, blood donors, and orthopedic patients with no records of mental disorders or suicidal behaviors and relatives of suicide attempters.

Basic sociodemographic features were collected for all the subjects. Psychiatric diagnoses were assessed using the Diagnostic Interview for Genetics Studies (DIGS) and the Mini International Neuropsychiatric Interview (MINI). Suicide behavior was assessed using the Suicidal Intent Scale (SIS) and the Risk Rescue Rating Scale (RRRS). Suicide ideation was examined with the Scale for Suicidal Ideation (SSI). The following scales validated in different languages were used for the investigation of the intermediate measures of suicidal behavior: the Life History of Aggression (LHA) interview, the Buss-Durkee Hostility Inventory (BDHI), the Spielberger State-Trait Anger Expression Inventory (STAXI), the Barratt Impulsivity Scale (BIS10), the Beck Depression Inventory (BDI), and the Beck Hopelessness Scale (BHS). The Childhood Trauma Questionnaire (CTQ), a retrospective measure of child abuse, and the CAGE questionnaire, for screening of alcohol problems, were also applied. All these variables were included in the dataset.

2.2. Variable Selection and Self-Organizing Maps. A selforganizing map (SOM) is a special kind of unsupervised neural network that consists of a bidimensional grid of units, each one characterized by a vector of the same dimension as the data. The grid can follow either a square or an hexagonal pattern, the latter being the most popular kind [11].

Training the network takes place iteratively using each training data point in two stages. In the first one, a best matching unit (BMU) is found so that its Euclidean distance 
TABLE 1: Variables with the highest value for the $d_{\text {SOM }}$ discriminant in the strongest peak of the SOM.

\begin{tabular}{lccccccc}
\hline Variable & $\widehat{c}$ & $\mu_{0}$ & $\mu_{1}$ & $\sigma_{0}$ & $\sigma_{1}$ & $d_{\text {SOM }}$ & $d_{f}$ \\
\hline Schizophrenia & 6.7072 & 0.1882 & 1.1908 & 1.2119 & 2.9261 & 1.5754 & 0.2423 \\
Bipolar & 5.8649 & 0.3417 & 1.1503 & 1.2263 & 2.9354 & 1.3271 & 0.1943 \\
Anxiety & 5.6541 & 0.8943 & 1.7824 & 1.5440 & 3.4972 & 0.9753 \\
Depression & 5.4166 & 0.8631 & 1.8613 & 1.3602 & 3.3084 & 0.9442 & 0.1762 \\
\hline
\end{tabular}

TABLE 2: Questions of the CAGE test.

\begin{tabular}{ll}
\hline & Question \\
\hline CAGE1 & $\begin{array}{l}\text { Have you ever felt you needed to cut down on your } \\
\text { drinking? }\end{array}$ \\
CAGE2 & Have people annoyed you by criticizing your drinking? \\
CAGE3 & $\begin{array}{l}\text { Have you ever felt guilty about drinking? } \\
\text { CAGE4 }\end{array}$ \\
& $\begin{array}{l}\text { Have you ever needed a drink as eye-opener in the } \\
\text { morning }(\cdots) ?\end{array}$ \\
\hline
\end{tabular}

TABLE 3: Variables with the highest value for the $d_{\mathrm{SOM}}$ discriminant at the second most outstanding cell.

\begin{tabular}{lccccccc}
\hline Variable & $\widehat{c}$ & $\mu_{0}$ & $\mu_{1}$ & $\sigma_{0}$ & $\sigma_{1}$ & $d_{\text {SOM }}$ & $d_{f}$ \\
\hline CAGE_TOT & 2.0134 & 0.1405 & 0.7163 & 0.6342 & 1.2751 & 0.9810 & 0.3016 \\
CAGE1 & 0.6422 & 0.0470 & 0.2175 & 0.2118 & 0.4129 & 0.9526 & 0.2729 \\
Score_CAGE & 0.6141 & 0.0389 & 0.2163 & 0.1936 & 0.4120 & 0.9496 & 0.2928 \\
CAGE2 & 0.6152 & 0.0471 & 0.2234 & 0.2120 & 0.4169 & 0.9034 & 0.2804 \\
\hline
\end{tabular}

TABLE 4: Questions of the BIS test involved in the third peak of the SOM. The subject rates the sentences according to the scale: (1) rarely/never, (2) occasionally, (3) often, and (4) almost always.

\begin{tabular}{ll}
\hline & Question \\
\hline bis_25 & I spend or charge more than I earn \\
bis_22 & I finish what I start \\
bis_2 & I do things without thinking \\
bis_6 & I am self-controlled \\
\hline
\end{tabular}

TABLE 5: Variables with the highest value for the $d_{\mathrm{SOM}}$ discriminant at the third most outstanding cell.

\begin{tabular}{lccccccc}
\hline Variable & $\widehat{c}$ & $\mu_{0}$ & $\mu_{1}$ & $\sigma_{0}$ & $\sigma_{1}$ & $d_{\text {SOM }}$ & $d_{f}$ \\
\hline bis_25 & 2.8591 & 1.3364 & 1.8414 & 0.7431 & 1.1184 & 0.8180 & 0.2713 \\
bis_22 & 1.7817 & 3.2021 & 2.6053 & 0.8455 & 1.1487 & 0.7123 & 0.2993 \\
bis_2 & 3.0516 & 1.7894 & 2.2266 & 0.8153 & 1.0474 & 0.6776 & 0.2347 \\
bis_6 & 1.8546 & 3.1744 & 2.2745 & 0.8897 & 1.0772 & 0.6710 & 0.4575 \\
\hline
\end{tabular}

(other distances have also been used. See [12] for an example with the information distance) to the data point is minimal. In the second stage, the centroid together with the BMU neighbors are moved in the direction of the data sample according to the rule

$$
\mathbf{m}_{i}^{(n+1)}=\mathbf{m}_{i}^{(n)}+\alpha^{(n)} h(w, i)\left(\mathbf{x}^{(n)}-\mathbf{m}_{i}^{(n)}\right),
$$

where $\mathbf{m}_{i}^{(n)}$ is the value of the $i$ th cell vector at instant $n$, $\alpha^{(n)}$ is the update rate, and $h(w, i)$ is the neighborhood kernel between the BMU $w$ and cell $i$.
TABle 6: Variables from the CTQ questionnaire. Possible answers are (1) never, (2) rarely, (3) occasionally, (4) often, and (5) almost always.

\begin{tabular}{ll}
\hline & Question \\
\hline CTQ_physnegl & $\begin{array}{l}\text { Physical neglect subscale } \\
\text { CTQ28_2 }\end{array}$ \\
I knew there was someone to take care of me and \\
protect me \\
CTQ28_7 & I felt loved \\
CTQ28_19 & People in my family felt close to each other \\
\hline
\end{tabular}

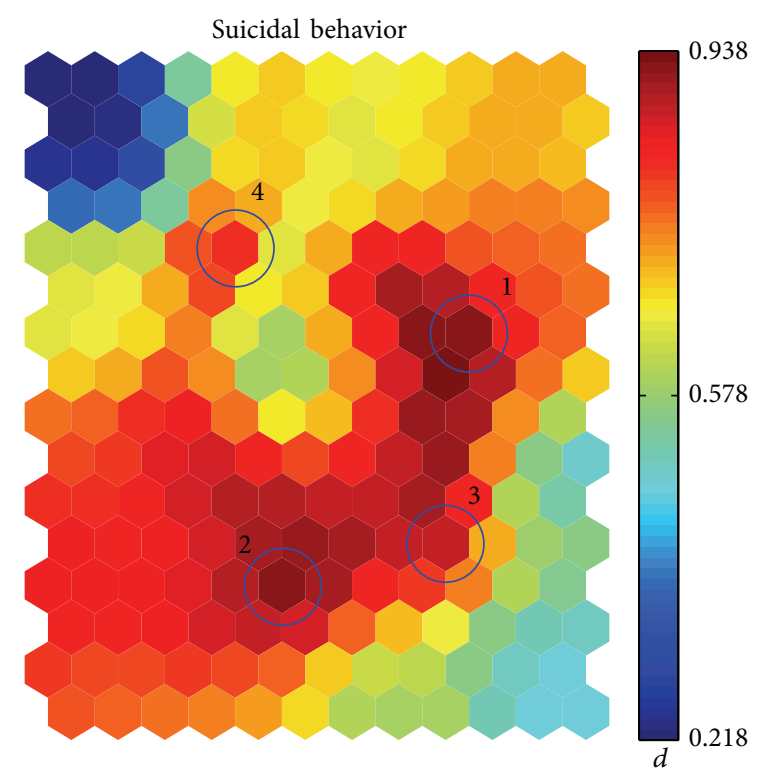

FIGURE 1: Maps obtained for the suicidal behavior auxiliary variable.

One of the main applications of SOMs is clustering, because a SOM consists of a set of centroids as an alternative to the set of centres provided by, for example, $k$-means. This ability for clustering is of interest for this particular application because we aim at identifying homogeneous groups of subjects characterized by different groups of variables and risk factors. Additionally, the two-dimensional layout of the centres allows for further interpretation of the clustering.

In order to rank the variables, according to classical statistics, one of the simplest ways is to use the Fisher discriminant [15]. For a binary decision problem, the Fischer discriminant of a variable $v$ is given by

$$
d_{F}(v)=\frac{\left|\mu_{1}(v)-\mu_{0}(v)\right|}{\sigma_{1}(v)+\sigma_{0}(v)} .
$$


TABLE 7: Variables with the highest value for the $d_{\text {SOM }}$ discriminant at the fourth most outstanding cell.

\begin{tabular}{lccccccc}
\hline Variable & $\widehat{c}$ & $\mu_{0}$ & $\mu_{1}$ & $\sigma_{0}$ & $\sigma_{1}$ & $d_{\text {SOM }}$ & $d_{f}$ \\
\hline CTQ_physnegl & 14.1902 & 8.9753 & 8.4325 & 3.7455 & 3.7974 & 0.6914 \\
CTQ28_2 & 1.7132 & 3.6056 & 3.7707 & 1.3350 & 1.4141 & 0.0720 \\
CTQ28_7 & 1.6115 & 3.3764 & 3.3393 & 1.3357 & 1.4242 & 0.6395 \\
CTQ28_19 & 1.7293 & 3.3107 & 3.1269 & 1.3010 & 1.4036 & 0.5847 & 0.0601 \\
\hline
\end{tabular}
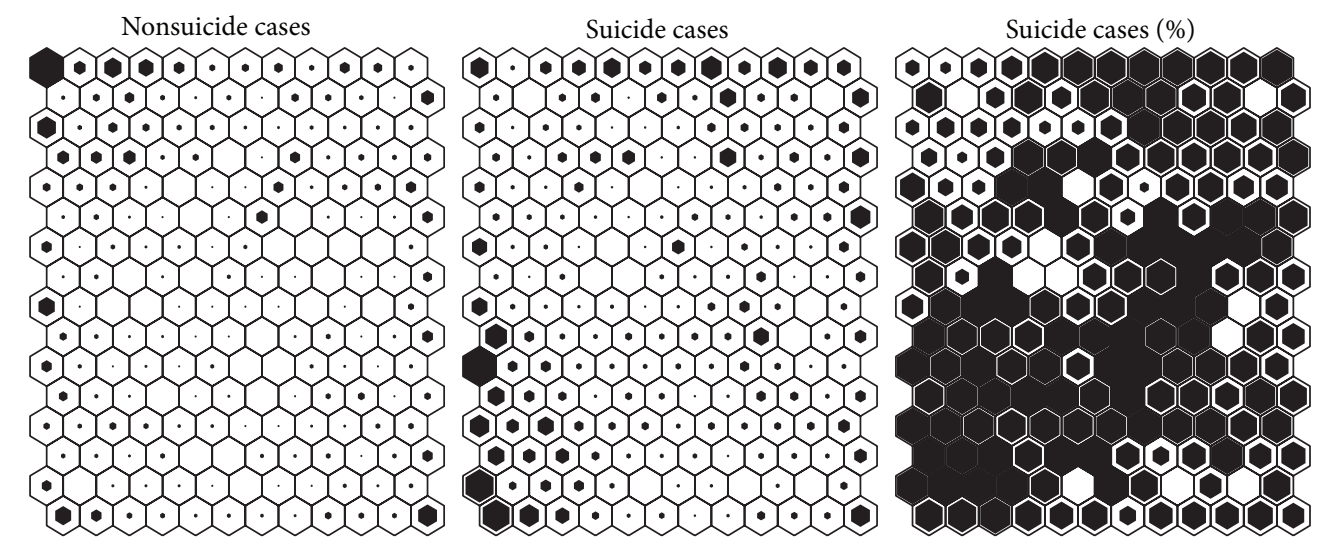

FIGURE 2: Histograms for the suicidal behavior variable. Left: negative samples; center: positive samples; right: percentage of positive samples in each cell.

Fisher's criterion is a linear discriminant. Hence, it is of limited application for variable selection in problems in which certain variables are strongly related to the auxiliary variable if the relationship is nonlinear. On the other hand, SOMs allow us to find risk factors by considering relationships among variables that can have a nonlinear nature. As mentioned previously, each centroid is updated by the data point that is closest at a given iteration. An auxiliary variable can be masked when building the data. However, its value is updated according to (1) and then visualized.

In order to use the SOM structure for variable ranking and selection, we look for the variables in which the distance between the value at the hottest unit in the map and the mean value of the negative class is the highest. In other words, we define a new discriminant, similar to Fisher's, given by the expression

$$
d_{\mathrm{SOM}}(v)=\frac{\left|\widehat{c}(v)-\mu_{0}(v)\right|}{\sigma_{1}(v)+\sigma_{0}(v)},
$$

where $\widehat{c}(v)$ is the value of the aforementioned centroid for variable $v$. Given that the value of the auxiliary variable of a given centroid is built based on the data matching that center, one can expect that the value of the variables in the hottest center-in terms of the auxiliary variable-is representative.

\section{Results and Discussion}

In the following, we apply the method described previously to the study of the variables most strongly related to suicidal behaviour. This variable indicates whether the subject has ever attempted suicide. There are 3213 positive and 1983 negative examples; the rest are undefined. We have trained an hexagonal SOM with $16 \times 12$ cells using the SOM Toolbox for Matlab [16]. A sequential training has been applied, with a linearly decreasing learning factor $\alpha^{(n)}$ that achieves convergence after running through the data for 4 times. A Gaussian neighbourhood function $h(w, i)$ has been used to train the SOM. The map is initialized in a deterministic way, setting the unit values to the eigenvectors of the covariance matrix of the data, that is, to the principal components. All the auxiliary variables in the dataset, that is, the ones referring to suicidal behaviour, such as SSI, RRRS, and SIS variablessee Section 2.1-are masked when finding the BMU, although their value is updated according to (1). Missing values are ignored in both the computation of the distances and the application of the update rule.

Once the map has been trained, we plot in Figure 1 the value of the suicidal behavior variable. In the figure, the most outstanding peaks or hot spots are highlighted in decreasing order of relevance. The criterion followed to choose these spots is descriptive rather than rigorous. This way, we stress the potential of this tool for building an interactive tool to assist the medical practice, in which the human intuition about the shape of the map is more important than a mathematical criterion for the detection of peaks. In particular, peaks 1, 2, and 3 have been chosen as the vertices of the $L$-shaped wide spot in the map; then, the 4 th peak is chosen as the most outstanding isolated spot.

Once the SOM is trained, each subject can be projected in the map by finding the closest cell in Euclidean distance, so that a histogram of correspondences is built. We show in Figure 2 the histograms of patients with and without a history of suicidal behavior, as well as the histogram with the percentage of subjects with suicide behavior per cell. Each bar in the left and middle 2-D histograms shows the amount 


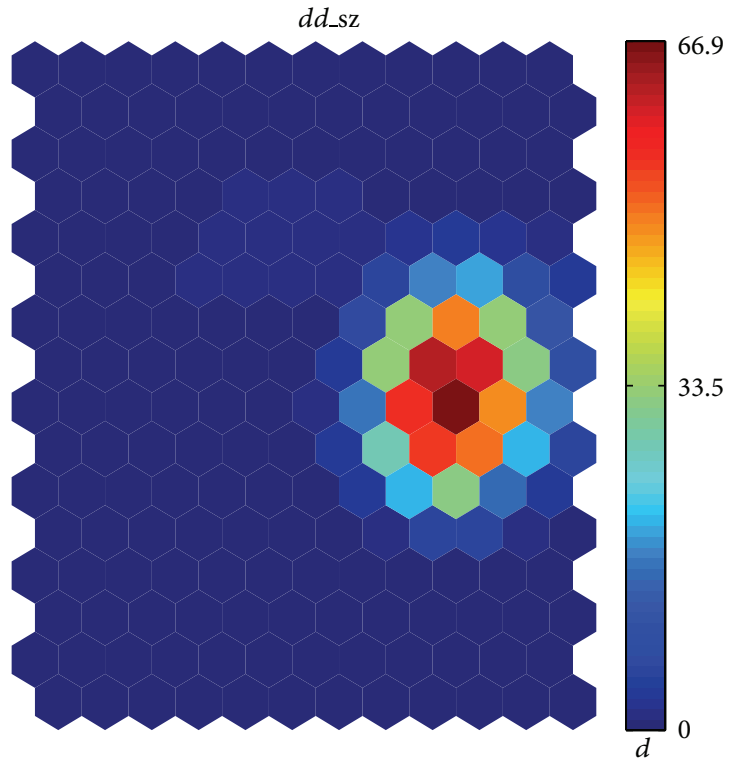

(a)

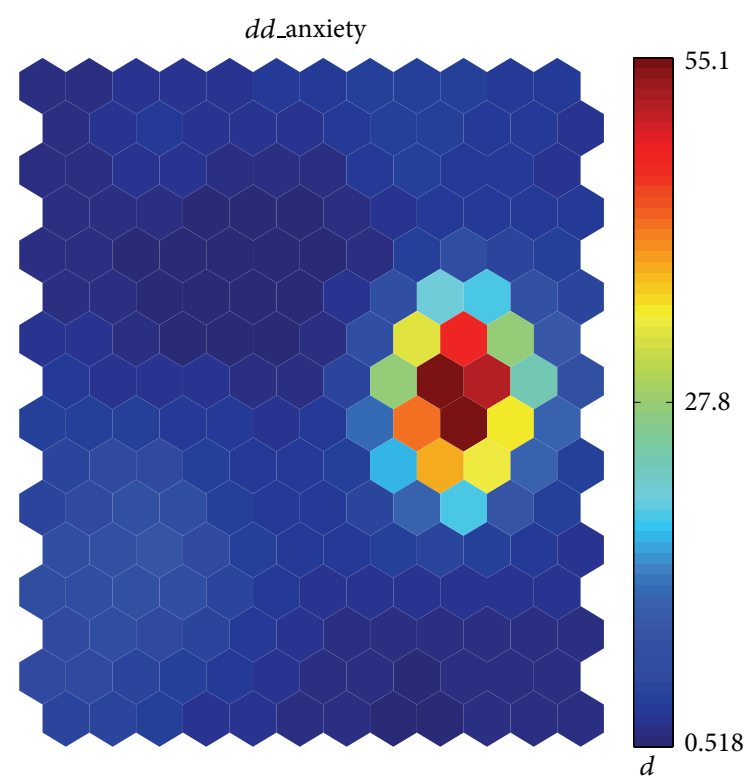

(c)

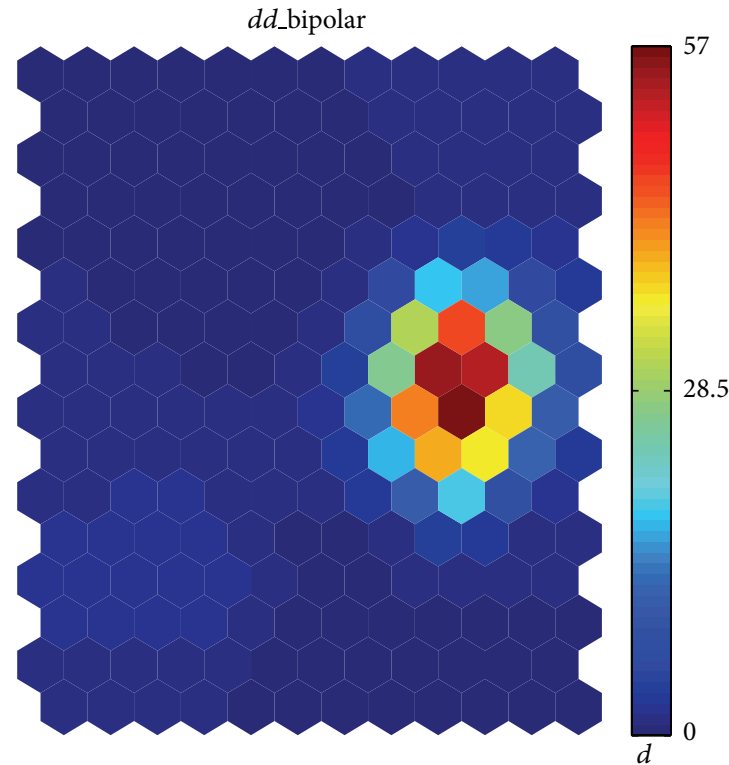

(b)

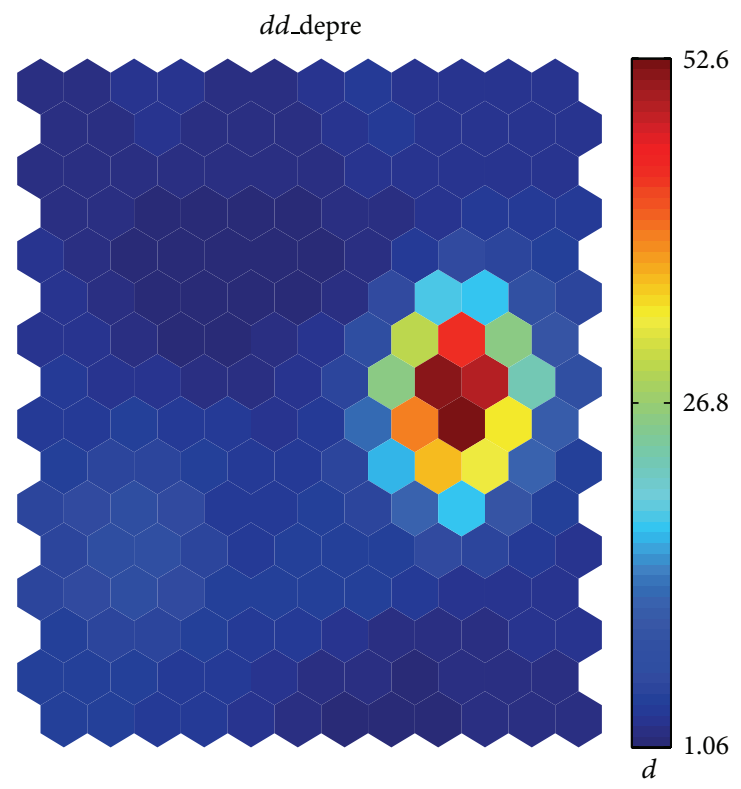

(d)

FIgURE 3: Distribution of the variables with highest $d_{\text {SOM }}$ value.

of data samples (subjects) that are closer to that cell than to any other. There is a high correspondence between the map with the variable values (Figure 1) and the histogram with the percentages (Figure 2, right).

We have computed the $d_{\text {SOM }}$ discriminant for the cell with the highest value of the suicidal behavior variable, that is, the one with the hottest color in Figure 1. Once the variables with more than $90 \%$ missing values are ignored, the list of variables with the highest value is shown in Table 1 . The most significant variables are related to mental disorder, which is consistent with the a priori medical knowledge that most mental disorders are associated with greater odds for attempting suicide. In contrast, the Fischer discriminant is unable to find these variables as relevant: these variables have been ranked by Fischer at locations 62, 98, 109, and 80 .

We show the map corresponding to these four most relevant variables in Figure 3. The high correlation between these maps and the one shown in Figure 1 is clear.

The second most outstanding cell according to Figure 1 gives the highest value of $d_{\text {SOM }}$ to variables CAGE_TOT, CAGE1, Score_CAGE, and CAGE2. The meanings of these variables are given by the answers to the CAGE questionnaire described in Table 2. Variables CAGE1 and CAGE2 are the answers to questions 1 and 2; CAGE_TOT is the summation 


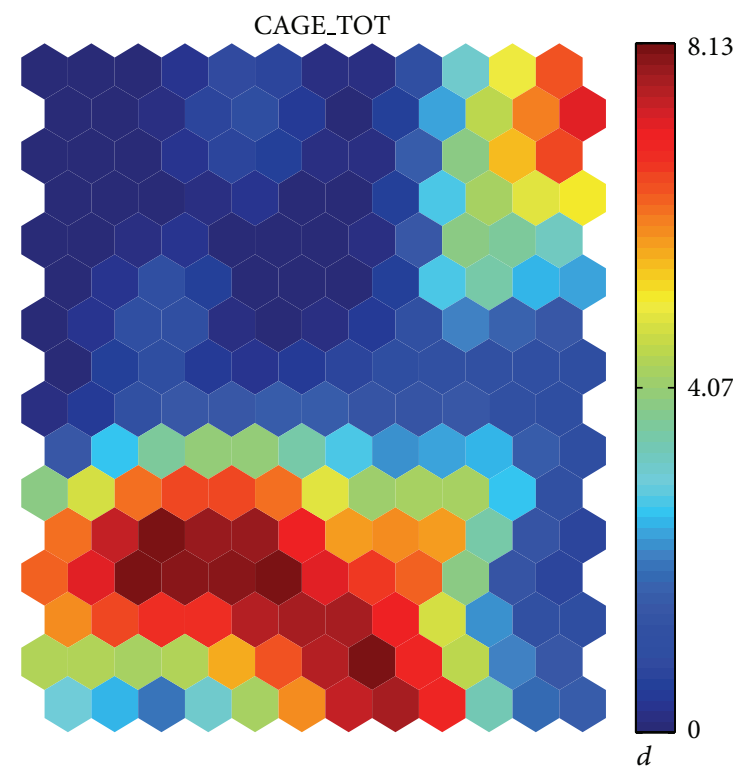

(a)

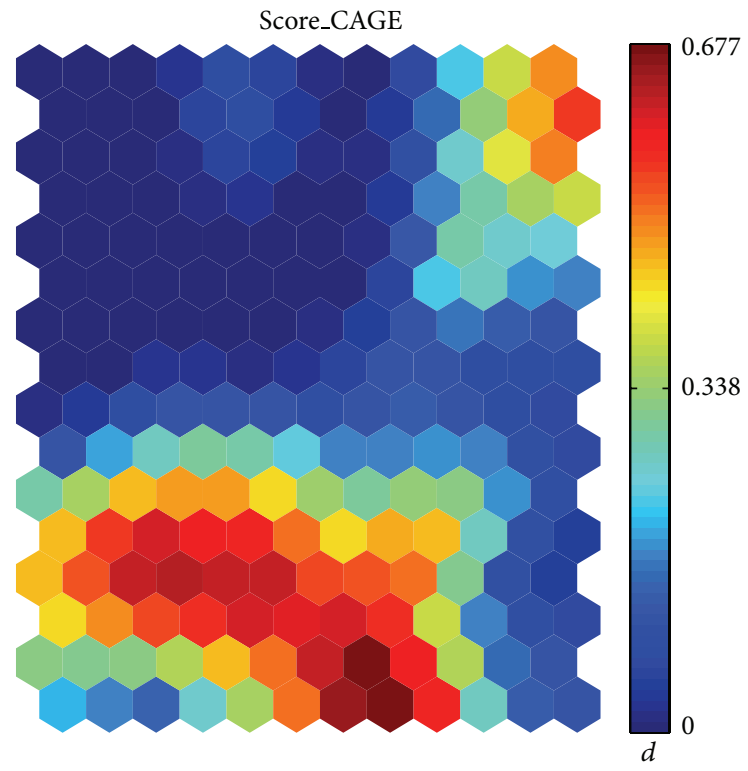

(c)

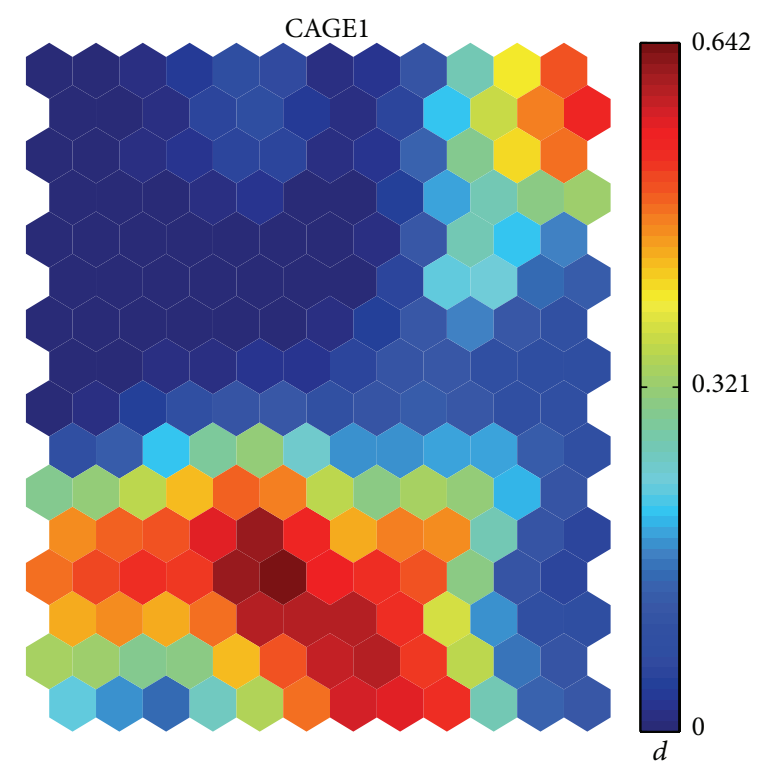

(b)

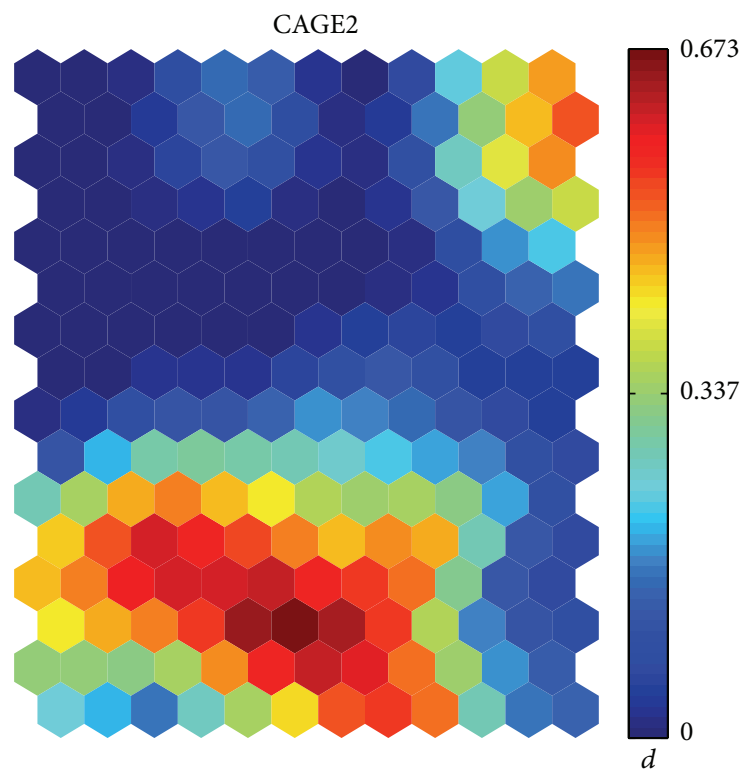

(d)

FIGURE 4: Distribution of the variables with the second highest $d_{\mathrm{SOM}}$ value in the suicidal behavior study.

of the four answers; SCORE_CAGE summarizes the total CAGE score in a yes-no category for alcohol problems. The distributions of these variables' values across the map are shown in Figure 4. The values of the discriminants are shown in Table 3.

The ranking of the most relevant variables related to the third peak of the map is headed by the questions of the BIS questionnaire described in Table 4. The statistics of the variables are shown in Table 5, and the corresponding maps are displayed in Figure 5. As the figure reveals, the maps corresponding to variables bis_22 and bis_6 show a negative correlation with respect to the map in Figure 1; that is, their values are low in the location of the third peak. This is due to the fact that questions bis_22 and bis_6 are formulated in a negative way; that is, agreement is related to the absence of impulsivity, unlike questions bis_25 and bis_2, in which a positive answer is related to impulsivity. According to (3), the discriminant can also be large by means of a cell value $c(v)$ lower than the average $\mu_{0}(v)$.

The exploration of the fourth peak reveals the presence of CTQ questions as the most relevant variables. Their meanings are described in Table 6; the statistics and maps are shown in Table 7 and Figure 6, respectively. Again, we observe a negative correlation in the maps corresponding to variables 


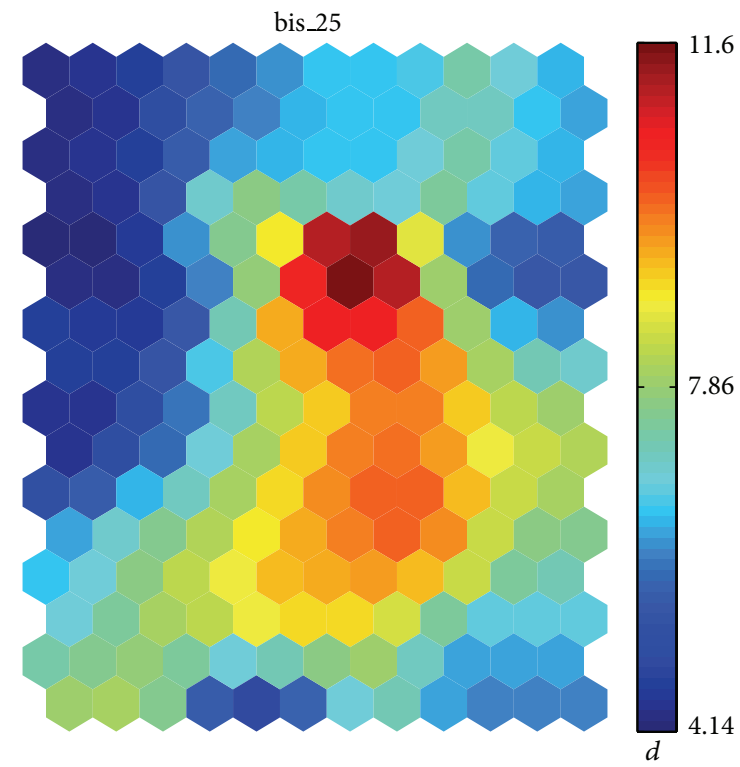

(a)

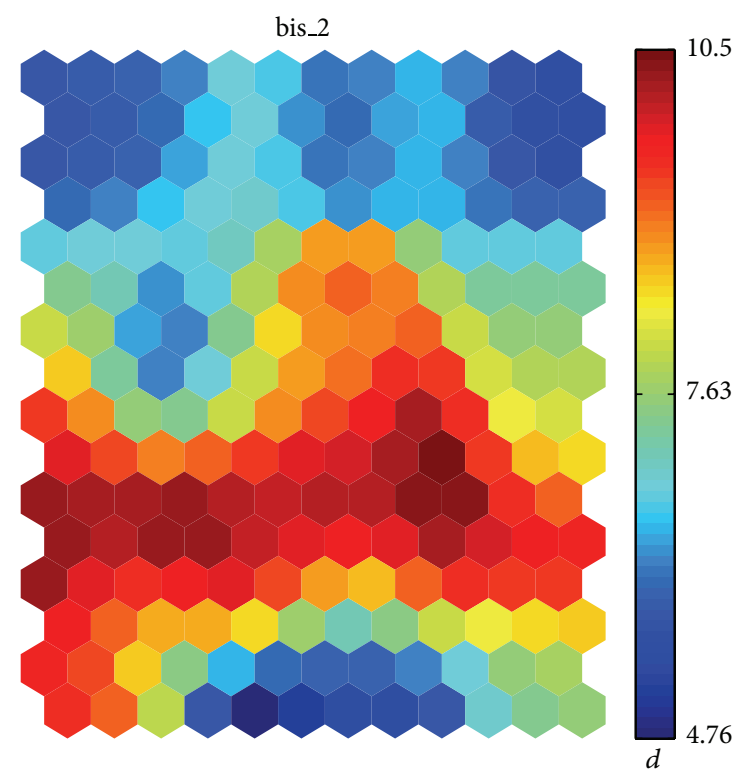

(c)

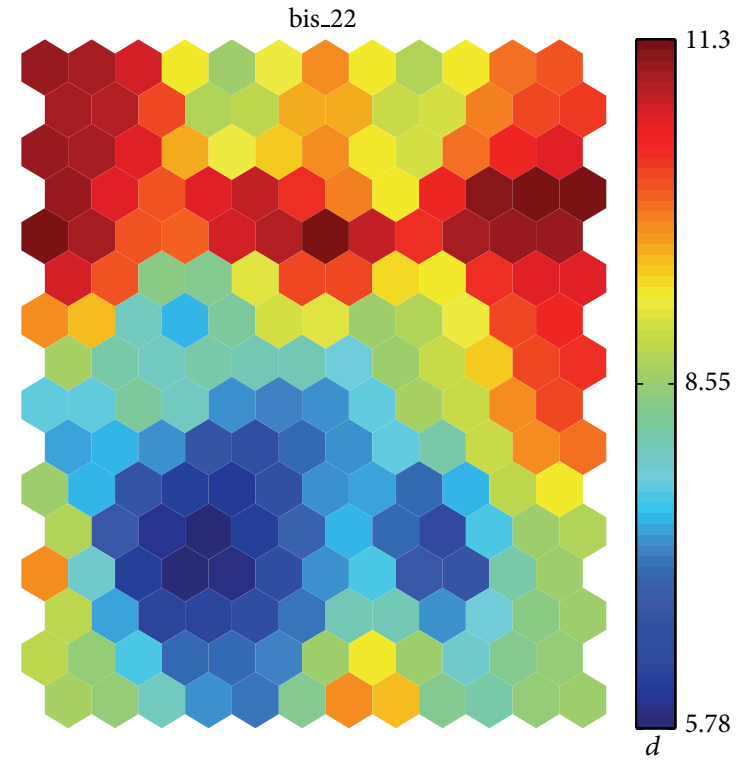

(b)

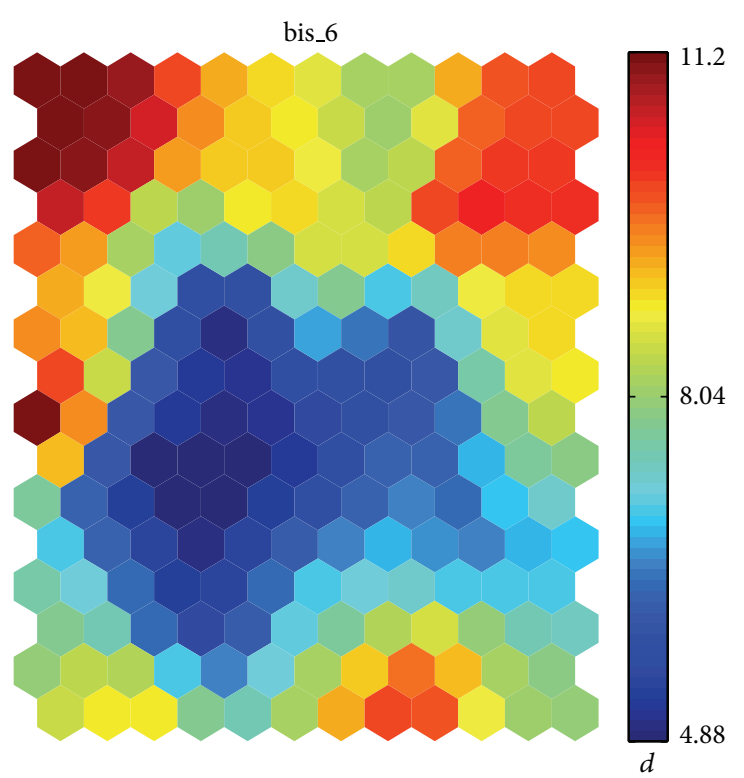

(d)

FIGURE 5: Distribution of the variables with the highest $d_{\mathrm{SOM}}$ at the third peak of the suicidal behavior SOM.

ctq28_2, ctq28_7, and ctq28_19, due to the fact that those questions are formulated in negative terms (a positive answer is opposite to neglect).

\section{Conclusions}

We have described a novel technique for variable selection based on self-organizing maps. The technique has been applied to a dataset containing socioeconomical, psychological, and clinical variables from a set of subjects that include suicide attempters. We make use of a discriminative criterion inspired by the Fisher discriminant to extract the variables most strongly related to the suicidal behavior. The map obtained for the suicidal behavior variable follows a complex structure with multiple peaks that can be interpreted as the existence of different groups or subpopulations of suicide attempters, with specific features. The study has revealed four groups of factors related to the four peaks observed in the SOM: mental disorders, alcoholism, impulsiveness, and childhood abuse, respectively. These results agree with current knowledge about the risk factors of suicide, which stresses the validity and usefulness of the described approach. The method can be extended to other problems of clinical interest in which heterogeneous risk factors are associated with a particular outcome in the population. The ability of the SOM to visualize the structure of high-dimensional 


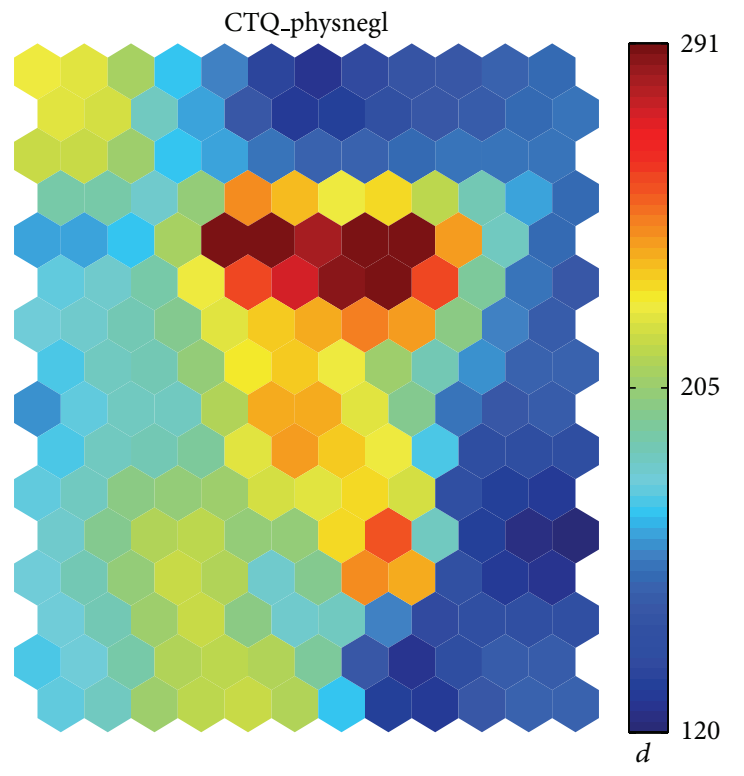

(a)

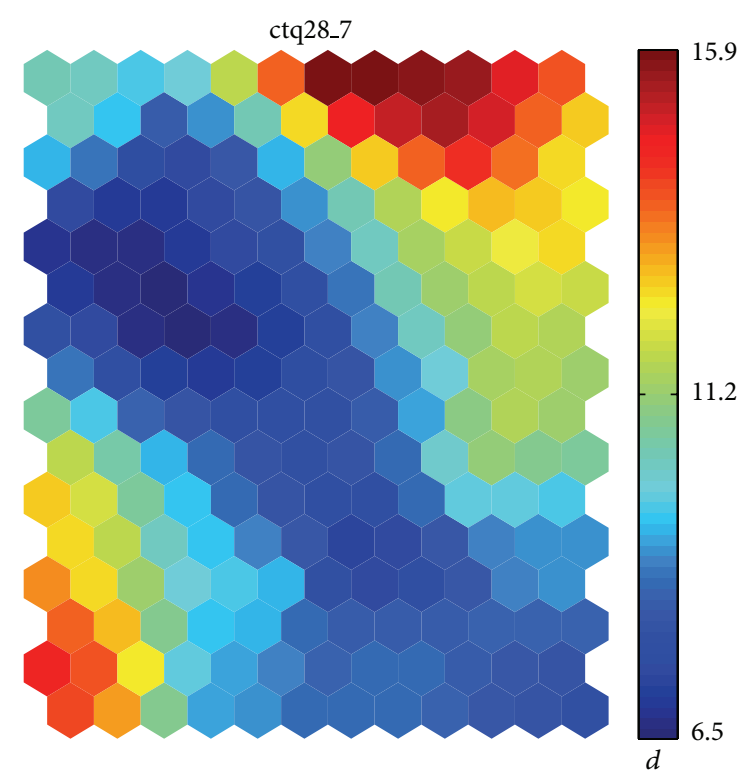

(c)

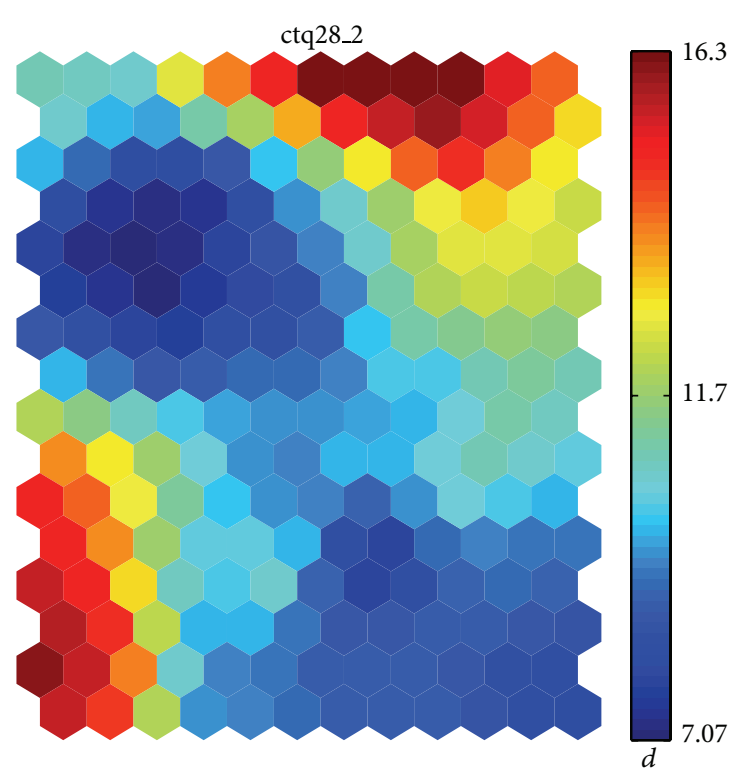

(b)

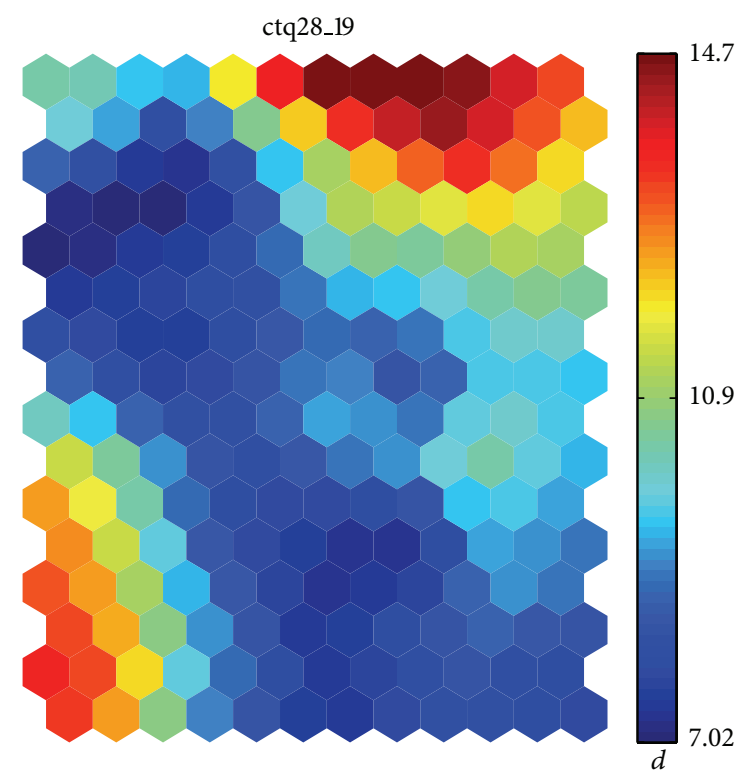

(d)

FIGURE 6: Distribution of the variables with the highest $d_{\text {SOM }}$ value at the fourth peak.

data makes the method suitable for developing tools for the clinical practice, where a deep understanding of the underlying statistics by the practitioner is not needed.

\section{Acknowledgments}

European Research Consortium for Suicide (EURECA). Individual members of the EURECA research team are as follows: Antonio Artes-Rodriguez (Department of Signal Theory and Communications, Carlos III University, Leganés, Madrid, Spain), Fuensanta Aroca (Instituto de Matemáticas UNAM. Mexico.), Nader Perroud (Department of Psychiatry,
University of Geneva Medical School, Switzerland), Pilar A Saiz (Department of Psychiatry, School of Medicine, University of Oviedo, Spain), Enrique Baca-Garcia, Jorge Lopez-Castroman, Hilario Blasco-Fontecilla (Department of Psychiatry, Villalba Mental Health Center, Spain), Marco Sarchiapone (Department of Psychiatry, University of Molise, Italy), Vladimir Carli (Department of Psychiatry, University of Molise, Italy), Philippe Courtet (Department of Emergency Psychiatry, CHRU Montpellier, Inserm U888, Montpellier, France), Isabelle Jaussent (Inserm U888, Montpellier, France), Sébastien Guillaume (Department of Emergency Psychiatry, CHRU Montpellier, Inserm U888, Montpellier, 
France) and Alain Malafosse (Department of Psychiatry, University of Geneva Medical School, Switzerland). This work was partially supported by the Spanish Ministry of Science and Innovation under Projects CSD2008-00010, TEC2009-14504-C02-01, and TEC2012-38800-C03-01, the Madrid Regional Government under the S2010/BMD-2422 Project, and PAPIIT-UNAM (Programa de Apoyo a Proyectos de Investigación e Innovación Tecnológica, Universidad Nacional Autónoma de México).

\section{References}

[1] J. J. Mann, A. Apter, J. Bertolote et al., "Suicide prevention strategies: a systematic review," The Journal of the American Medical Association, vol. 294, no. 16, pp. 2064-2074, 2005.

[2] G. C. Patton, C. Coffey, S. M. Sawyer et al., "Global patterns of mortality in young people: a systematic analysis of population health data," The Lancet, vol. 374, no. 9693, pp. 881-892, 2009.

[3] F. Jollant, F. Bellivier, M. Leboyer et al., "Impaired decision making in suicide attempters," The American Journal of Psychiatry, vol. 162, no. 2, pp. 304-310, 2005.

[4] M. K. Nock, G. Borges, E. J. Bromet, C. B. Cha, R. C. Kessler, and S. Lee, "Suicide and suicidal behavior," Epidemiologic Reviews, vol. 30, no. 1, pp. 133-154, 2008.

[5] M. A. Oquendo, D. Currier, and J. J. Mann, "Prospective studies of suicidal behavior in major depressive and bipolar disorders: what is the evidence for predictive risk factors?" Acta Psychiatrica Scandinavica, vol. 114, no. 3, pp. 151-158, 2006.

[6] D. Delgado-Gomez, H. Blasco-Fontecilla, A. A. Alegria, T. Legido-Gil, A. Artes-Rodriguez, and E. Baca-Garcia, "Improving the accuracy of suicide attempter classification," Artificial Intelligence in Medicine, vol. 52, no. 3, pp. 165-168, 2011.

[7] J. Leiva-Murillo, A. A. Rodrguez, and E. Baca-Garca, "Visualization and prediction of disease interactions with continuoustime hidden markov models," 2011.

[8] A. Antos, L. Devroye, and L. Györfi, "Lower bounds for Bayes error estimation," IEEE Transactions on Pattern Analysis and Machine Intelligence, vol. 21, no. 7, pp. 643-645, 1999.

[9] D. H. Wolpert, "The lack of a priori distinctions between learning algorithms," Neural Computation, vol. 8, no. 7, pp. 13411390, 1996.

[10] I. Guyon and A. Elissee, "An introduction to variable and feature selection," Journal of Machine Learning Research, vol. 3, pp. 11571182, 2003.

[11] T. Kohonen, Self-Organizing Maps, Springer, Berlin, Germany, 3rd edition, 2001.

[12] J. Peltonen and S. Kaski, "Discriminative components of data," IEEE Transactions on Neural Networks, vol. 16, no. 1, pp. 68-83, 2005.

[13] J. Lopez-Castroman, M. Perez-Rodriguez, I. Jaussent et al., "Distinguishing the relevant features of frequent suicide attempters," Journal of Psychiatric Research, vol. 45, no. 5, pp. 619-625, 2011.

[14] M. M. Silverman, A. L. Berman, N. D. Sanddal, P. W. O’Carroll, and T. E. Joiner, "Rebuilding the tower of babel: a revised nomenclature for the study of suicide and suicidal behaviors part 2: suicide-related ideations, communications, and behaviors," Suicide and Life-Threatening Behavior, vol. 37, no. 3, pp. 264-277, 2007.

[15] K. Fukunaga, Introduction to Statistical Pattern Recognition, Academic Press, New York, NY, USA, 1990.
[16] J. Vesanto, J. Himberg, E. Alhoniemi, J. Parhankan-gas, S. Team, and L. Oy, "Som toolbox for matlab," Techn. Ber., Helsinki University of Technology, 2000. 


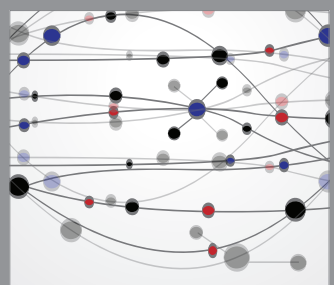

The Scientific World Journal
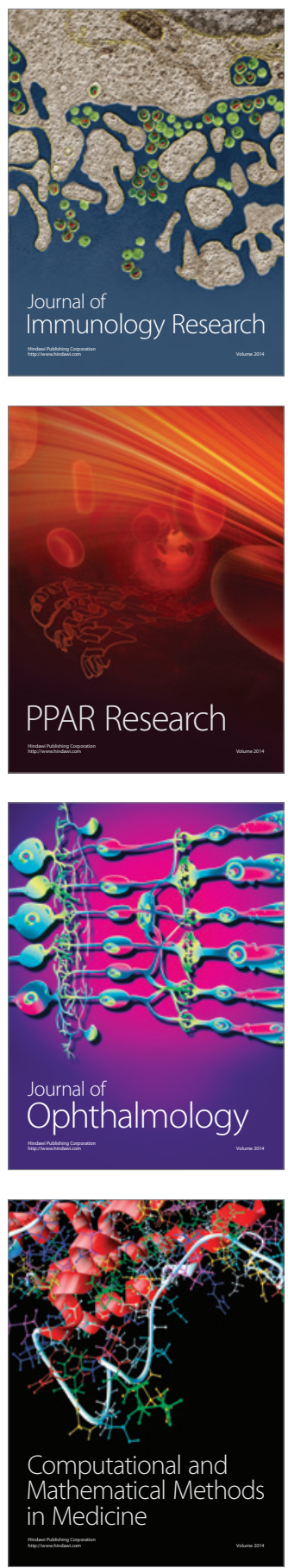

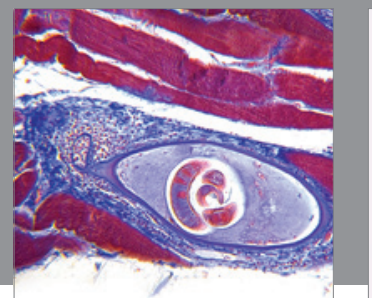

Gastroenterology

Research and Practice
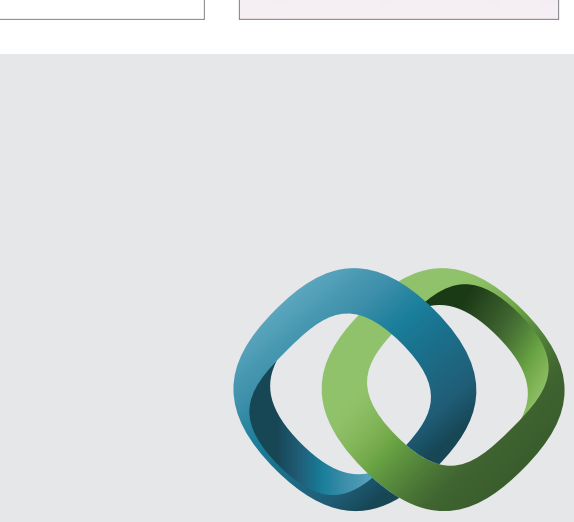

\section{Hindawi}

Submit your manuscripts at

http://www.hindawi.com
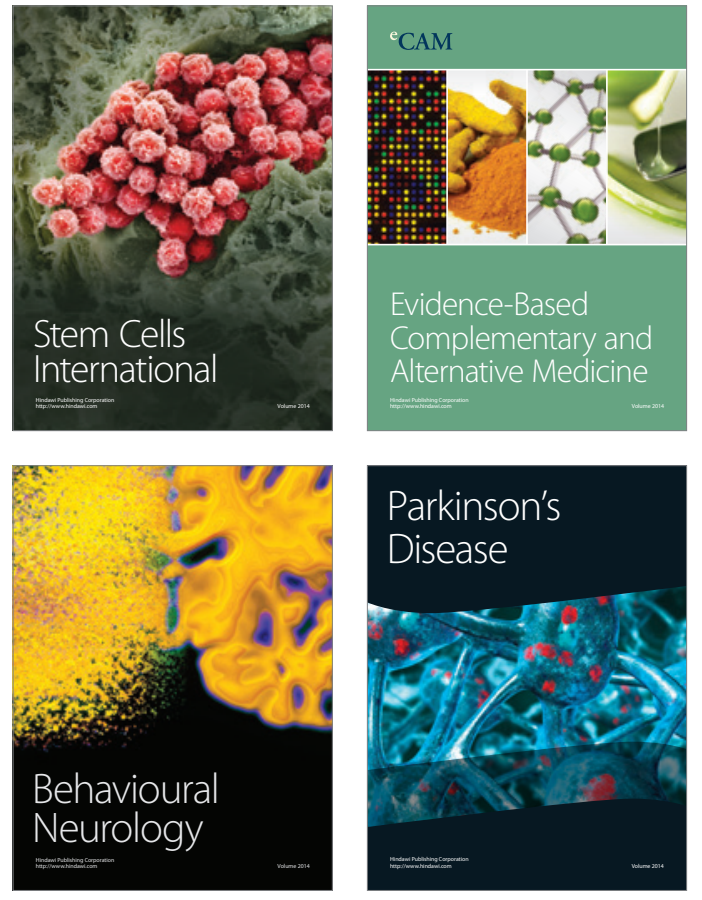
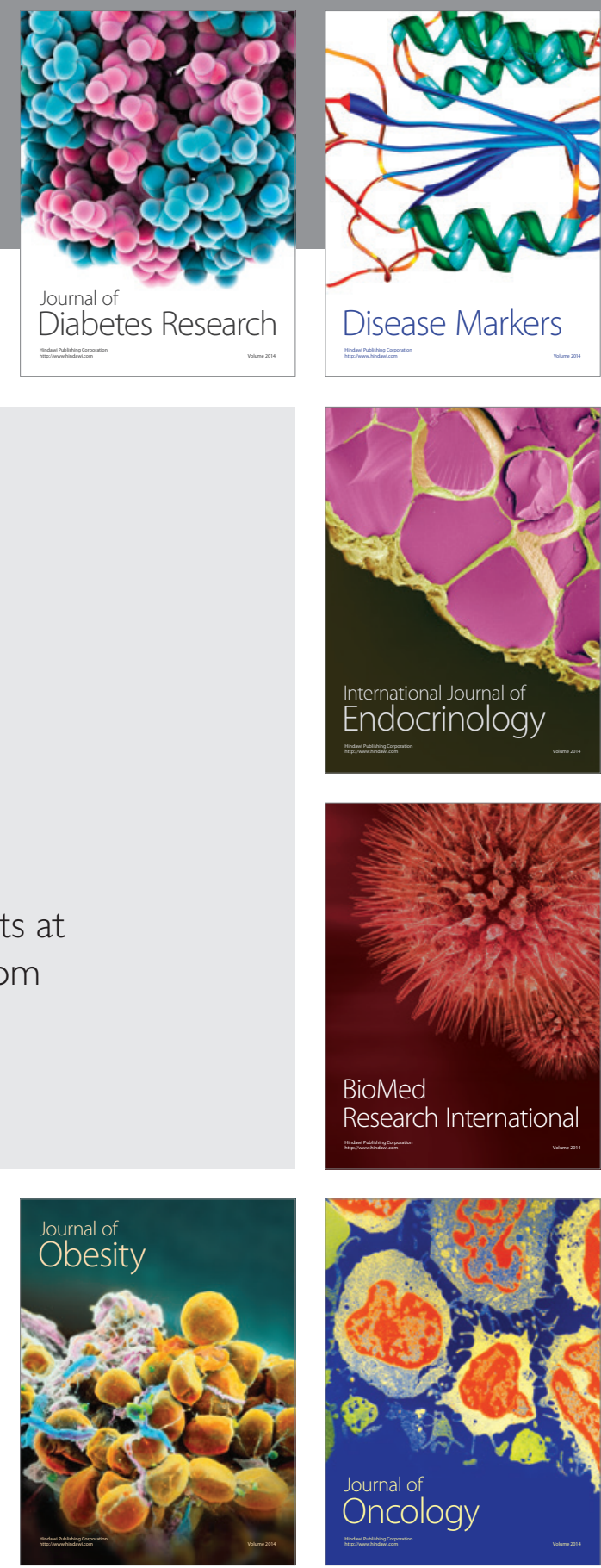

Disease Markers
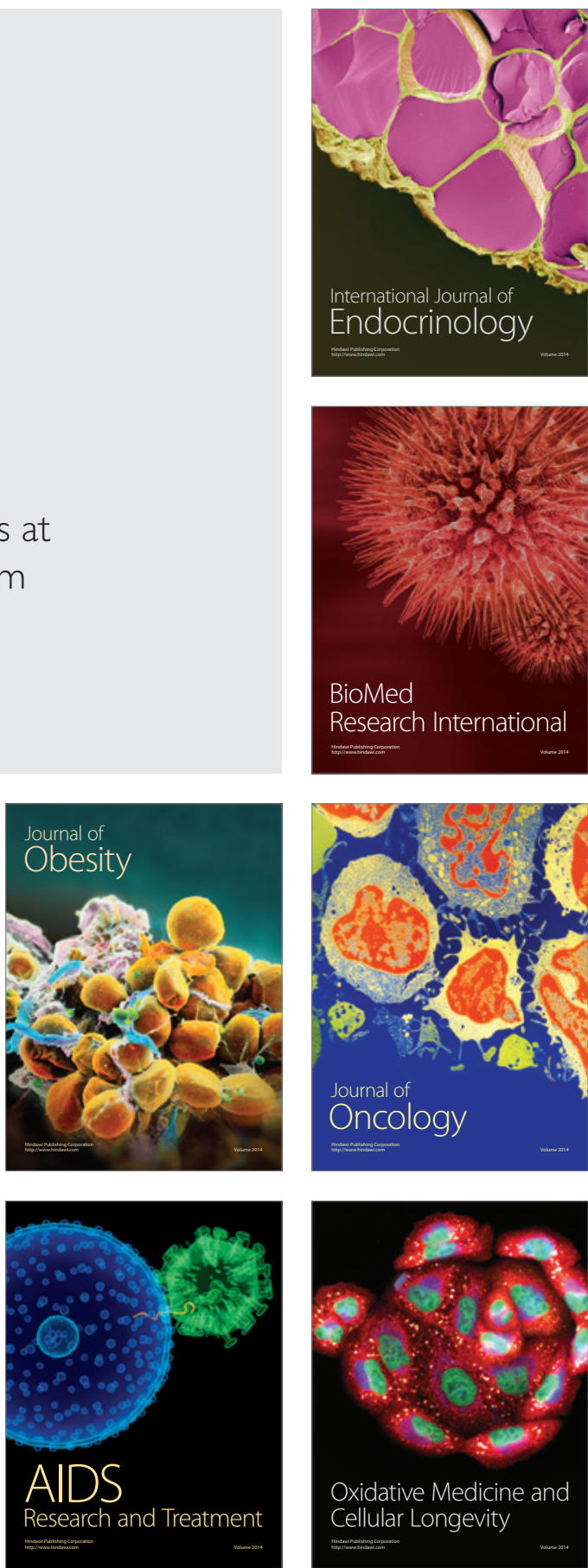